\title{
HETEROGENEIDAD ESPACIAL DE LAS COMUNIDADES FITOPLANCTONICAS DE LA ALBUFERA DE VALENCIA
}

\author{
M.R. Miracle; M.P. García y E. Vicente \\ Departamentos de Ecología y Microbiología Facultad de Ciencias Biologias. Universidad de Valencia \\ Palabras clave: Freshwater phytoplankton, hypertrophic coastal lagoon, Albufera of Valencia. (Spain).
}

\author{
ABSTRACT \\ SPATIAI. HETEROGENEITY OF THE PHYTOPLANKTONIC COMMUNITIES OF THE ALBUFERA \\ OF VALENCIA.
}

The plankton of the Albufera of Valencia, an hypertrophic coastal lagoon, is dominated by Cyanobacteria, which in most cases constitute the $80 \%$ of the phytoplankton. The analysis of the samples, taken in different zones of the lagoon, has made apparent the extraordinary population densities reached by the phytoplankton. It maintains, on the average, a density around one million of individuals per $\mathrm{ml}$, which roughly corresponds to a biomass of $200 \mathrm{mg} / \mathrm{land}$ toa chlorophyll $a$ concentration of $0,4 \mathrm{mg} / \mathrm{l}$. The mean specific pigment contents of Albufera phytoplankron is around the $2 \%$ of chlorophyll $a$ per unit of biomass, which is relatively low compared with other lakes but frequent when the plankton is dominated by Cyanobacteria.

In the plankton of the Albufera 95 species of algae have been identified: 32 Cyanobacteria, 38 Clorophyceae, 26 Diatoms, 6 Euglenophyceae, 2 Crytophyceae and 1 Dinoflagellate. The statistical analysis of algal counts demostrates the existence of three different communities of species corresponding to the distinct zones of the lagoon as follows:

1.- A community constituted by several closely associated species of Lyngbya and Oscillatoria which dominates in the most part of the lagoon (localities 1 to 4 and 8 ).

2.- A second one made by the association of Cyclotella glomerata. Nitzscbia palea and Scenedesmus quadricauda, which characterizes rhe zone near Barranc of Massanassa (locality 7).

3.- A third community associated to the last mentioned one constituted mainly by Stephanodiscal hantaschit Cbrosocous planctonicus and Merimopedia tenuessma located at the Northern zone to the West of Barranc of Massanassa (localities $S$ and 6 ).

\section{INTRODUCCION}

La Albufera de Valencia es una laguna litoral enmarcada por la zona de arrozales de la huerta valenciana. La influencia en ella de la actividad humana es extraordinaria, pues su flujo de agua está regulado por los procesos del cultivo del arroz y es además el sumidero general de los vertidos urbanos correspondientes a más de medio millón de habitantes, así como de los procedentes de las aproximadamente cinco mil industrias enclavadas a su alrededor. La acción reiterada y creciente de vertidos tan contaminantes, ha convertido recientemente a esta laguna en un sistema hipertrófico extremo, cuya densidad fitoplanctónica es de las mayores registradas en el mundo.

Dada la gran extensión e importancia natural y

Limnética 1: 20- 31 (1984)

(C) Asociación Española de Limnología, Madrid. Spain socio-económica de la Albufera de Valencia, extraña el que no haya ni una sola publicación dedicada al fitoplancton, exceptuando menguadas listas de unas pocas especies puestas como ejemplos en trabajos más amplios, dedicados a otros componentes biológicos (Pardo, 1942; Blanco, 1957). El fitoplancton de la Albufera ha variado enormemente en los últimos años, pero cualquier comparación con base científica resulta dificil, al no disponer de estudios anteriores que permitirían seguir su evolución a lo largo del tiempo.

El presente trabajo pretende dar una idea de las actuales características del fitoplancton de la Albufera, pero pòr la gran extensión de esta laguna y el muy distinto origen de los aportes de agua que recibe, se ha 
hecho necesario practicar un estudio en sus diferentes zonas, teniendo especial cuidado en la elección de una serie de puntos de muestreo que sean máximamente representativos. Con ello, se intenta dar una visión general del fitoplancton de toda la laguna, tanto e $\mathrm{n}$ su composición específica y estructura de la comunidad como desde el puntode vista de su densidad, biomasa y concentración de pigmentos fotosintéticos. Se insiste además en las variaciones temporales que se observan en los distinto puntos, que son debidas principalmente a los cambios de flujo y renovación de las aguas del lago.

\section{MATERIAL Y METODOS}

El lago.- La Albufera es una laguna litoral somera (profundidad media $0,75 \mathrm{~m}$ ), situada al sur de la ciudad de Valencia ocupandola parte central, hoy muy mermada (2.200 Ha.), de una vasta zona húmeda (más de 23.000 Ha.) que antaño se extendia entre los deltas de los ríos Turia y Jucar. Establece comunicación con el mar a través de tres canales o golas que atraviesan el cordón litoral que la separa del mar, y en los que el caudal de agua vertido en cada momento se controla por la acción de compuertas a fín de adecuar el nivel de la laguna a las exigencias del cultivo del arroz. Los aportes de agua se hacen a través de las numerosas acequias y barrancos que afluyen en todo su contorno, destacando el barranco de Chiva (acequia de Massanassa) y los de el Hondo y la Berenguera (acequias de Overa y Dreta, Roselló, 1979). Las aguas de la laguna, más saladas en épocas pretéritas, hoy son de tipo oligohalino e hipertrófico.

Metodos.- El muestreo de la Albufera se ha efectuado durante un recorrido sucesivo por ocho puntos del lago que pretende cubrir las distintas zonas.de la laguna. Se efectuaron tres campañas correspondiente a los meses de julio, septiembre y noviembre.

La transparencia del agua se determinó con la ayuda del disco de Secchi, calculando posteriormente el coeficiente de extinción para las distintas estaciones, y muestreos. Mediciones directas de la penetracion de la energía luminosa se llevaron a cabo con un qiiantameter Crump mod. 550. Simultameamente, se midieron otros parámetros físico-químicos cuyos resultados pueden consultarse en Oltra y Miracle (1984).
Para la cuantificación de los pigmentos fotosintéticos, muestras de $50 \mathrm{ml}$ de agua se filtraron a través de discos de fibra de vidrio Whatman GF/F, extrayéndose los pigmentos durante 12-24 horas a -20Qen $5 \mathrm{mlde}$ una mezcla de acetona $90 \%$ y DMSO en proporción 1:1 (Shoaf y Lium, 1976), y su contenido en clorofilas, feofitinas y carotenoides se valoró espectrofotométricamente según Lorenzen (1967) y Strickiand y Parsons (1972).

Para el recuento del fitoplancton, muestras de agua de las diferentes estaciones, tomadas a $0,5 \mathrm{~m}$. de profundidad por bombeo peristáltico, fueron fijadas con lugol. Su recuento se efectuó por observación directa de los organismos con ayuda de un microscopio invertido (1.250 aumentos), después de sedimentar volúmenes de 1 a $3 \mathrm{ml}$ en cubetas cilíndricas (Vollenweider, 1974). El número de campos ópticos contados fué el necesario para totalizar un mínimo de 1.000 individuos por muestra en muestras duplicadas. Se efectuó además un barrido de toda la cubeta paro detectar la presencia de especies poco frecuentes. Las algas filamentosas se expresaron como número de filamentos y las especies coloniales como número de agrupaciones o colonias. Los cálculos de la biomasa fueron realizados multiplicando el número de individuos por su volumen correspondiente, calculado basándose en su forma geométrica y las dimensiones medias de las poblaciones presentes en cada muestra.

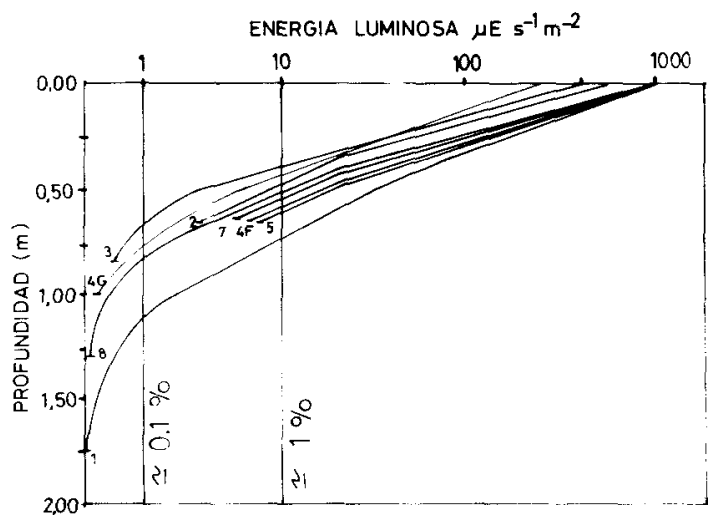

Figura 1. Penerración de la luz cn el agua en los distintos puntos de muestreo ( 1 a 8). Nótese como. por término medio, a un metro de profundidad llega menos del $1 \%$ de la luz incidente.

Extincrion of light in the water at thedifferent sampling stations ( 1 to 8 ) Notice that ir arrives generaly at 1 mof depth less than $1 \%$ of incident light. 


\section{RESULTADOS Y DISCUSION}

1.-Pigmentos fotosintéticos.

Las diferentes magnitudes para medir el plancton vegetal (pigmentos, $\mathrm{n}^{\mathrm{o}}$ de individuos y biomasa) presentan unas relaciones muy claras entre sí, que depende de la comunidad fitoplanctónica de que se trate, interrelacionada a su vez con las características del medio.

En la Albufera de Valencia, la concentración de clorofila a rebasa en todo momento $\operatorname{los} 100 \mathrm{mg} / \mathrm{m}^{3}$, valor que resultó ser de los mayores obtenidos en un estudio de 100 embalses españoles (Margalef $\boldsymbol{e t}$ al. 1977). En julio, prácticamente todos los puntos de muestreo, y en septiembre, la mayoría de ellos, alcanzaron valores superiores a los $350 \mathrm{mg} / \mathrm{m}^{2}$ (Tabla I). El valor máximo registrado fué de $646 \mathrm{mg} / \mathrm{m}^{3}$, correspondiente al mes de septiembre y a la estación 7, momento y localidad en el que se constató un crecimiento destacado de diatomeas, algunas de las cuales se hacen dominantes.

Un valor de $350 \mathrm{mg} / \mathrm{m}^{2}$ de clorofila $a$ es suficiente para absorber la práctica totalidad de la energía luminosa utilizable a la profundidad de $1 \mathrm{~m}$, pero el hecho de que esta cifra a menudo se rebase (Tabla I)

revela que a menos de un metro ya ha sido capturada toda la luz incidente (Fig. 1), no encontrándose activa la clorofila de las algas que se hallen por debajo de dicha profundidad, y sólo mediante la circulación del agua puede entrar de nuevo en la zona iluminada. Es muy probable que la Albufera, incluso en sus zonas más someras, utilice toda la luz disponible, alcanzando su máximo potencial de producción primaria.

Estas concentraciones de clorofila tan elevadas, que

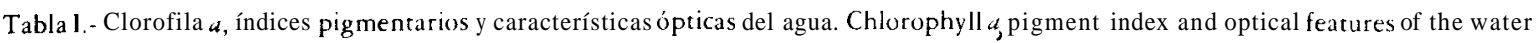

\begin{tabular}{|c|c|c|c|c|c|c|}
\hline Est. & Fecha & $\underset{\left(\mathrm{mg} / \mathrm{m}^{3}\right)}{\operatorname{Clor}}$ & $\frac{\text { Feof. }}{\text { Clor }}$ & $\frac{D_{430}}{D_{665}}$ & $\mathrm{~S}_{(\mathrm{m})} \mathrm{hi}$ & $\begin{array}{l}\text { Prot. } \\
1 \%\left(\mathrm{~m}^{\mathrm{j}}\right)^{z}\end{array}$ \\
\hline \multirow{3}{*}{1} & $27-07-82$ & 451,23 & 3,42 & 1,92 & 0,24 & 0,65 \\
\hline & $21-09-82$ & 217,00 & 16,60 & 1,80 & 0,30 & 0,81 \\
\hline & $4-11-82$ & 114,81 & 4,94 & 1,92 & 0,22 & 0,60 \\
\hline \multirow{3}{*}{2} & $27-07-82$ & 349,77 & 2,98 & 1,96 & 0,24 & 1,96 \\
\hline & $21-09-82$ & 186,90 & 1,95 & 1,87 & 0,32 & 1,87 \\
\hline & $4-11-82$ & 144,18 & 0,32 & 1,89 & 0,25 & 1,39 \\
\hline \multirow{3}{*}{3} & $27-07-82$ & 307,05 & 0,10 & 2,01 & 0,28 & 0,76 \\
\hline & $21-09-82$ & 368,46 & 0,10 & 1,96 & 0,28 & 0,76 \\
\hline & $4-11-82$ & 170,88 & 2,69 & 1,95 & 0,19 & 0,51 \\
\hline \multirow{3}{*}{4} & $27-07-82$ & 429,87 & 0,10 & 1,99 & 0,22 & 0,60 \\
\hline & $21-09-82$ & 347,10 & 10,86 & 1,83 & 0,28 & 0,76 \\
\hline & $4-11-82$ & 293,70 & 5,92 & 2,06 & 0,21 & 0,57 \\
\hline \multirow{3}{*}{5} & $27-07-82$ & 491,28 & 9,32 & 2,02 & 0,21 & 0,57 \\
\hline & $21-09-82$ & 277,68 & 25,13 & 1,92 & 0,31 & 0,84 \\
\hline & $4-11-82$ & 253,65 & 6,82 & 2,02 & 0,21 & 0,57 \\
\hline \multirow{3}{*}{6} & $27-07-82$ & 368,46 & 10,24 & 2,00 & 0,25 & 0,68 \\
\hline & $21-09-82$ & 437,88 & 16,04 & 1,95 & 0,20 & 0,54 \\
\hline & $4-11-82$ & 210,93 & 8,12 & 1,98 & 0,21 & 0,57 \\
\hline \multirow{3}{*}{7} & $27-07-82$ & 440,55 & 15,62 & 1,94 & 0,20 & 0,54 \\
\hline & $21-09-82$ & 646,14 & 17,95 & 2,05 & 0,15 & 0,41 \\
\hline & $4-11-82$ & 165,54 & 10,10 & 2,06 & 0,21 & 0,57 \\
\hline \multirow{3}{*}{8} & $27-07-82$ & 485,05 & 3,92 & 2,01 & 0,25 & 0,68 \\
\hline & $21-09-82$ & 368,46 & 0,10 & 1,90 & 0,30 & 0,81 \\
\hline & $4-11-82$ & 205,59 & 2,61 & 2.04 & 0,20 & 0,54 \\
\hline
\end{tabular}


sitúan a la Albufera en un grado de hipertrofia extrema, son de adquisición reciente. En la monografía de Dafauce (1975) sobre dicha laguna se hace patente que, eri la pasada década, hubo un incremento anual progresivo del contenido de clorofila, aumento que continua en la actualidad. A modo ilustrativo podemos señalar que en un punto de muestreo, próximo al denominado por nosotros estación 8 , los máximos de concentración de clorofila $\boldsymbol{a}$ en los años 1972, 1973 y 1974 fueron respectivamente 20,44 y $78 \mathrm{mg} / \mathrm{m}^{4}$.

La figura 2 representa la distribución de las desviaciones a la medida del contenido en clorofila $\boldsymbol{a}$ del agua. Se observa que las poblaciones fitoplanctónicas fluctuan desfasadas en unas zonas del lago respecto a otras. En Julio, resalta un gran crecimiento algal en el foco de contaminación de Font Nova (est. 5) que se extiende por toda la parte central, situándose los mínimos en la zona Sur principalmente. En septiembre se dió un gran máximo en el Barranc de Massanassa (est. 7), con mínimos en la zona SE yen la correspondiente al máximo anterior (estación 5). En noviembre, el área de máxima concentracibn se desplazó hacia el oeste, abarcando la Foia (est. 4) y Font Nova, mientras que toda la zona SE, muy afectada por las inundaciones de la Ribera Baja del Júcar, acaecidas el 20 de octubre de 1982, presentaba los valores mínimos.

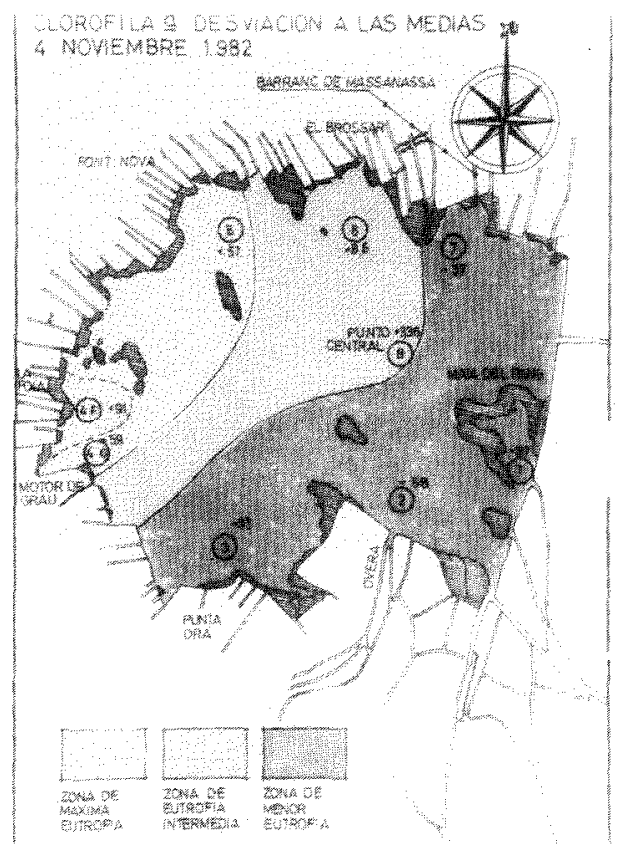

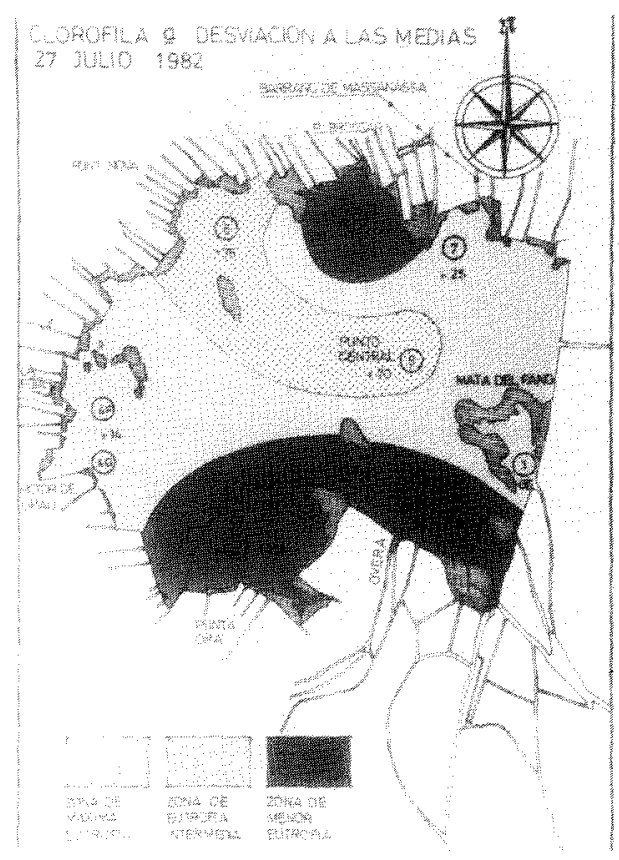

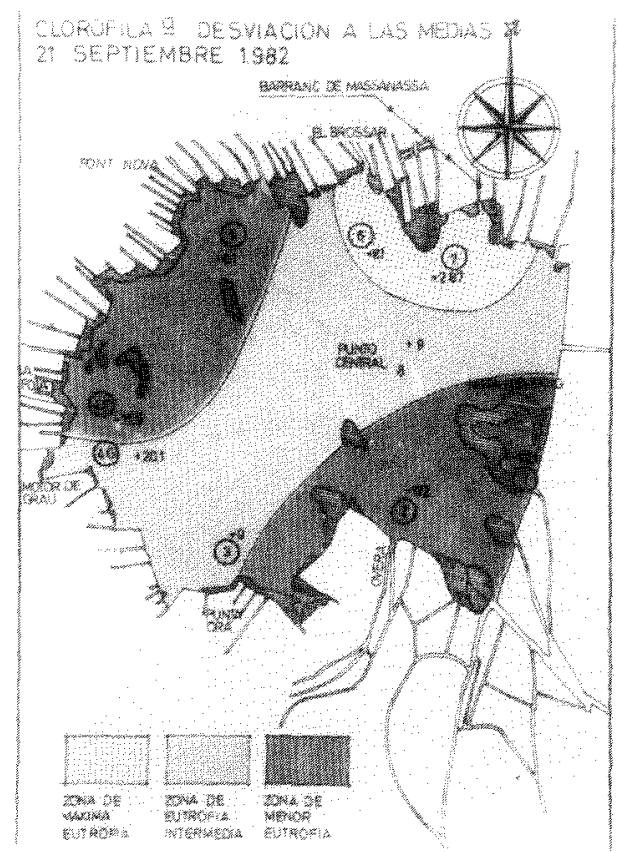

Figura 2- Distribución de las desviaciones a la media de la concentración de clorofila $a$ en la Albufera correspondientes a las fechas del 27 de Julio, 21 de Septiembre y 4 de Noviembre, respectivamente.

Distribution of the desviations to the mean of the chlorophyll a concentrations in the dates of July 27 , September 21 and November 4 , respectively. 
Las relaciones de la concentración de clorofila $\boldsymbol{a}$ con el número de individuos y con la biomasa fitoplanctónica (Tabla Il) están representadas en el Fig. 3, donde se trazó la recta de regresión lineal de sus valores transformados logarítmicamente. Estas relaciones son altamente significativas y bastante lineales, incluso con los valores originales sin transformación, oscilando el contenido de clorofila a por individuo entre 0,4 y 0,5 pg y ajustándose mucho el contenido de clorofila $a$ por unidad de biomasa al $2 \%$.

En la representación de la relación de clorofila $a / n^{0}$ de indiviudos (figura $3 \mathrm{~A}$ ) se observa que este contenido es, en general, $m$ ás bajo que $0^{\prime} 4$ $\mathrm{pg} /$ individuo en las muestras de verano, y superior a
0'5 pg/individuo en las de Noviembre. En el estudio del fitoplancton de los embalses españoles (Margalef, 1977) se obtuvo algo parecido, esto es, el contenido de clorofila a por célula en las épocas de mezcla era significativamente superior al de verano o época de estratificación. En el caso de la relación de la clorofila a con la biomasa (Fig. 3B), el cociente varía al revés de lo dicho para la relación clorofila $a / \mathrm{n}^{\circ}$ de individuos, es decir, presenta valores superiores a los medios, que resultan ser del $2 \%$, en julio e inferiores en noviembre. Esta aparente contradicción es explicable debido al menor tamaño de las células filamentosas y coloniales en las condiciones de alta temperatura de verano, lo que hace que el contenido de clorofila a por

Tabla II.- Densidad y biomasa del fitoplancton, contenido en clorofila u e índice de diversidad de Shannon. Phytoplanktonic density and biomass, chlorophyll u contents and Shannon's diversity index.

\begin{tabular}{|c|c|c|c|c|c|c|c|}
\hline Est. & Fecha & \multicolumn{2}{|c|}{$\begin{array}{l}\text { Fitoplancton } \\
(\mathrm{ind} / \mu \mathrm{l})(\mathrm{mg} / \mathrm{l})\end{array}$} & $\frac{\text { Biornasa }}{\text { indiv. }}$ & $\frac{\text { Clor. a }}{\text { indiv }}$ & 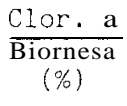 & $\begin{array}{c}\text { Indice } \\
\text { Diversidad } \\
\text { (bits/ind) }\end{array}$ \\
\hline \multirow{3}{*}{1} & $27-07-82$ & 1330 & 153,82 & 115,7 & 0,34 & 0,29 & 2,58 \\
\hline & $21-09-32$ & 697 & 136,23 & 195,5 & 0,40 & 0,20 & 3,13 \\
\hline & $4-11-82$ & 296 & 81,84 & 276,5 & 0,40 & 0,14 & 2,70 \\
\hline \multirow{3}{*}{2} & $27-07-82$ & 1237 & 158,66 & 128,3 & 0,28 & 0,22 & 2,61 \\
\hline & $21-09-82$ & 472 & 95,85 & 203,1 & 0,41 & 0,20 & 2,99 \\
\hline & $4-11-82$ & 300 & 88,77 & 295,9 & 0,49 & 0,16 & 2,71 \\
\hline \multirow{3}{*}{3} & $27-07-82$ & 999 & 113,53 & 113,6 & 0,30 & 0,26 & 2,31 \\
\hline & $21-09-82$ & 805 & 193,28 & $240, \mathrm{t}$ & 0,46 & 0,19 & 3,19 \\
\hline & $4-11-82$ & 342 & $84, \hat{09}$ & 247,6 & 0,51 & 0,20 & 2,77 \\
\hline \multirow{3}{*}{4} & $27-07-82$ & 1302 & 127,37 & 97,8 & 0,33 & 0,34 & 2,14 \\
\hline & $21-09-82$ & 867 & 244,20 & 281,7 & 0,44 & 0,16 & 3,33 \\
\hline & $4-11-32$ & 559 & 156,97 & 262,9 & 0,54 & 0,21 & 3,02 \\
\hline \multirow{3}{*}{5} & $27-07-82$ & 1891 & 229,91 & 121,6 & 0,27 & 0,22 & 1,88 \\
\hline & $21-09-32$ & 580 & 144,42 & 249,0 & 0,39 & 0,16 & 3,61 \\
\hline & $4-11-82$ & 491 & 121,42 & 247,3 & 0,54 & 0,22 & 2,35 \\
\hline \multirow{3}{*}{6} & $27-07-82$ & 1383 & 193,50 & 159,9 & 0,28 & 0,20 & 2,38 \\
\hline & $21-09-82$ & 1171 & 276,32 & 236,0 & 0,42 & 0,18 & 2,87 \\
\hline & $4-11-82$ & 422 & 112,04 & 265,5 & 0,53 & 0,20 & 3,58 \\
\hline \multirow{3}{*}{7} & $27-07-82$ & 569 & 88,14 & 154,9 & 0,83 & 0,54 & 3,31 \\
\hline & $21-09-82$ & 1399 & 261,22 & 186,7 & 0,53 & 0,28 & 3,00 \\
\hline & $4-11-82$ & 271 & 88.62 & 327,0 & 0,65 & 0,20 & 3,45 \\
\hline \multirow{3}{*}{8} & $2 \vec{\imath}-0 \vec{i}-82$ & 1647 & 201,36 & 122,3 & 0,29 & 0,24 & 2,40 \\
\hline & $21-09-82$ & 1196 & 261,94 & 219,0 & 0,31 & 0,14 & 3,01 \\
\hline & $4-11-32$ & 451 & 125,76 & 278,8 & 0,46 & 0,17 & 2,31 \\
\hline
\end{tabular}




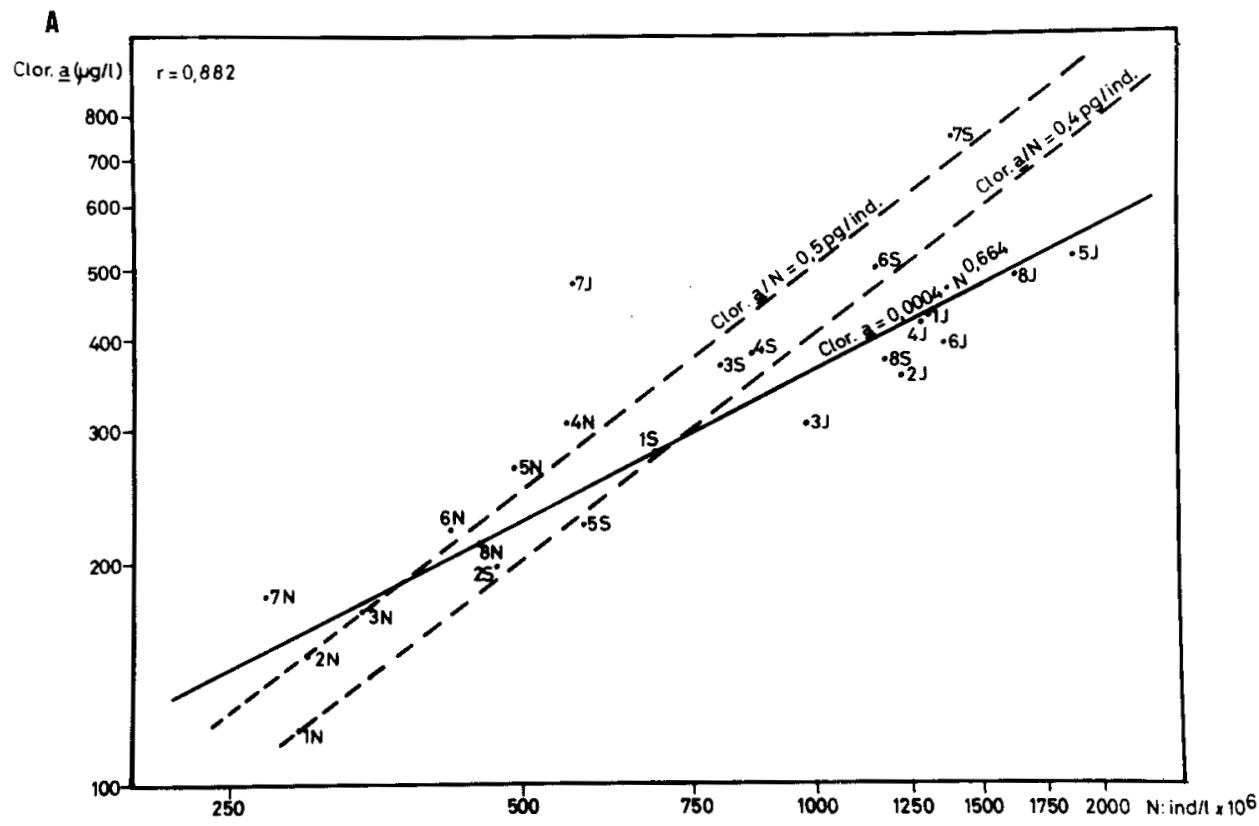

\section{B}

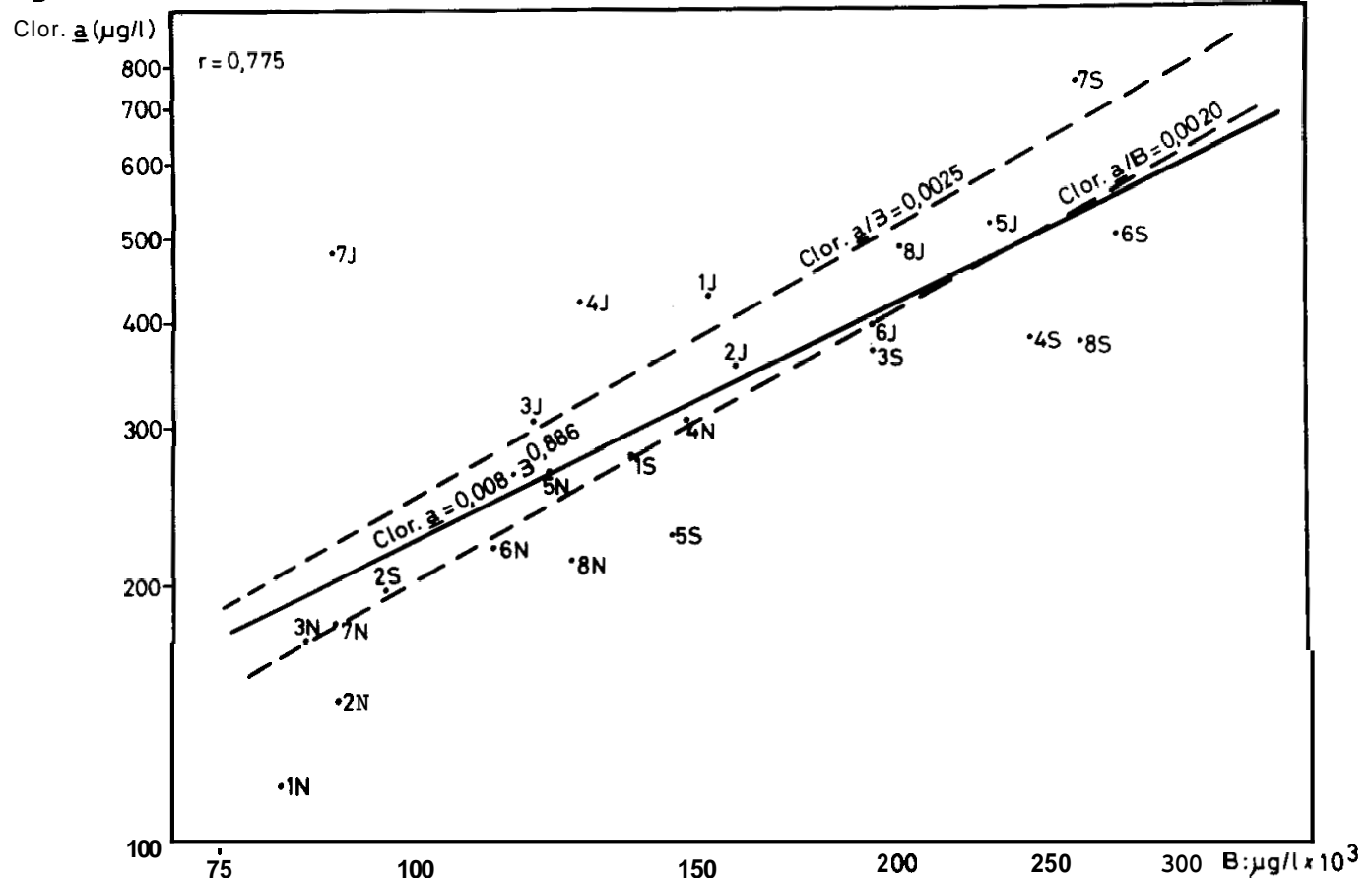

Figura 3 - Relación de la concentración de clorofila a con el número de individuos (A) y con la biomasa (B). (Elnúmeroindica la localidad y la letra el mes a que pertenecen los datos).

Relationshipbetween chlorophylla concentration with the number of individuals (A) and the biomass (B). Sampling stations and months are indicated by a number and a letter, respectively 
individuo sea menor, pero en cambio referido a unidad de biomasa sea mayor, ya que estas condiciones inducen el crecimiento de poblaciones fitoplantónicas de menor tamaño celular medio.

Se observa en la estación 7, e n la que el dominio de las cianobacterias no es tan absoluto, el contenido en clorofila $a$ individuos y el contenido específico son más altos que las medias (Tabla II), lo que concuerda perfectamente con otros estudios (Margalef, 1977) en el hecho de que las comunidades fitoplanctónicas dominad; por cianobacterias son las que muestran valores más bajos para estas relaciones.

El índice pigmentario de Margalef (D430/D665) oscila en la Albufera entre 1'8 y 2'06 (Tabla I), valor bajo como corresponde a aguas claramente eutróficas, manteniéndose muy constante en el tiempo y el espacio, contrariamente a lo que sucede en otros lagos y embalses como los representados también en la figura 4, en la que se comparan los índices obtenidos para la Albufera frente a los presentados por un lago oligotrófico, Banyoles y un embalse eutrófico, La Minilla (Planas, 1973; Toja, 1980). Según consta en la literatura sobre el tema, este índice alcanza valores entre 2.4 y 3,6 en lagos oligotróficos y en los lagos eutróficos se situa entre 1'4 y 2'4 (Margalef, 1980).

Otro índice pigmentario de interés en la calidad de las aguas es el cociente feofitinas/clorofila $a$, que representa la cantidad de clorofila degradada frente a la clorofila activa. Este índice (Tabla I) es alto en la Albufera, y comparativamente más alto en las zonas más contaminadas (estaciones 5, 6 y 7).

2.- Composición específica y estructura de la comunidad fitoplanctónica.

Se han identificado en la Albufera de Valencia, 95 especies de algas plantónicas, presentándose en la Tabla III una relación de las más frecuentes, indicando su importancia en el plancton. También se ha considerado in grupo de bacterias heterótrofas: grupo Planctomyou Blastocaulis, por su incidencia numérica en algunos de los recuentos efectuados.La composición fitoplantónica del lago se distribuye en 22 especies de cianobacterias, 38 de cloroficeas, 26 de diatomeas, 6 de euglenales, 2 criptoficeas $y]$ dinoflagelada; no obstante, son tan solo unas pocas especies de cianobacterias las que dominan el plancton, destacando las especies de los géneros Lynglya y Oscilla. toria. Estas cianobacterias filamentosas se encuentran adaptadas preferentemente a la vida bentónica y su

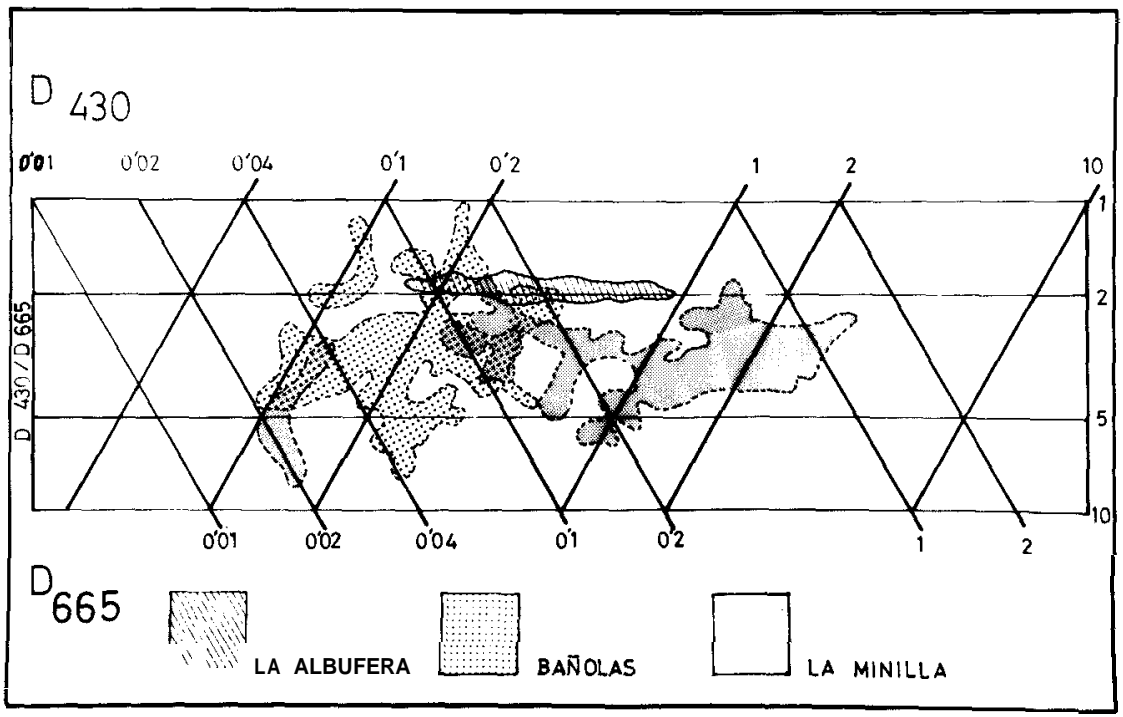

Figura í - Variación de los prgmentos torosintéricos eii la Albutera comparada con la del lago de Banyoles y el embalse de la Minilla. Las áreas engloban los distintos resultados durante el período de estudio, en la Albufera y de un ciclo anual,en los otros lugares. Los diferentes puntos se situan con referencia a dos escalas logarítmicas que se cruzan con un ángulo de $120^{\circ}$, que expresan las densidades ópticas a $430 \mathrm{y}$ $665 \mathrm{nni}$ del estracto de pigmentos. Las líneas horizontales corresponden a los valores del índice pigmentario $\mathrm{D}_{430} / \mathrm{D}_{6069}$

Variations of photosynthetic pigments in the Albufera compared with that of Banyoles lake and la Minilla reservoir. The areas enclose the different resulrs during rhe study period in rhe Albufera and during the annual cycle in the other places. The different points are refered to two logarithmic scales, forming an angle of $120^{\circ}$ which represent the optical densities at 430 and $665 \mathrm{~nm}$. of the pigment extracts Horizontal lines correspond ro the $\mathrm{D}_{4 \leqslant 0} / \mathrm{D}_{6,69}$ pigment ratio. 


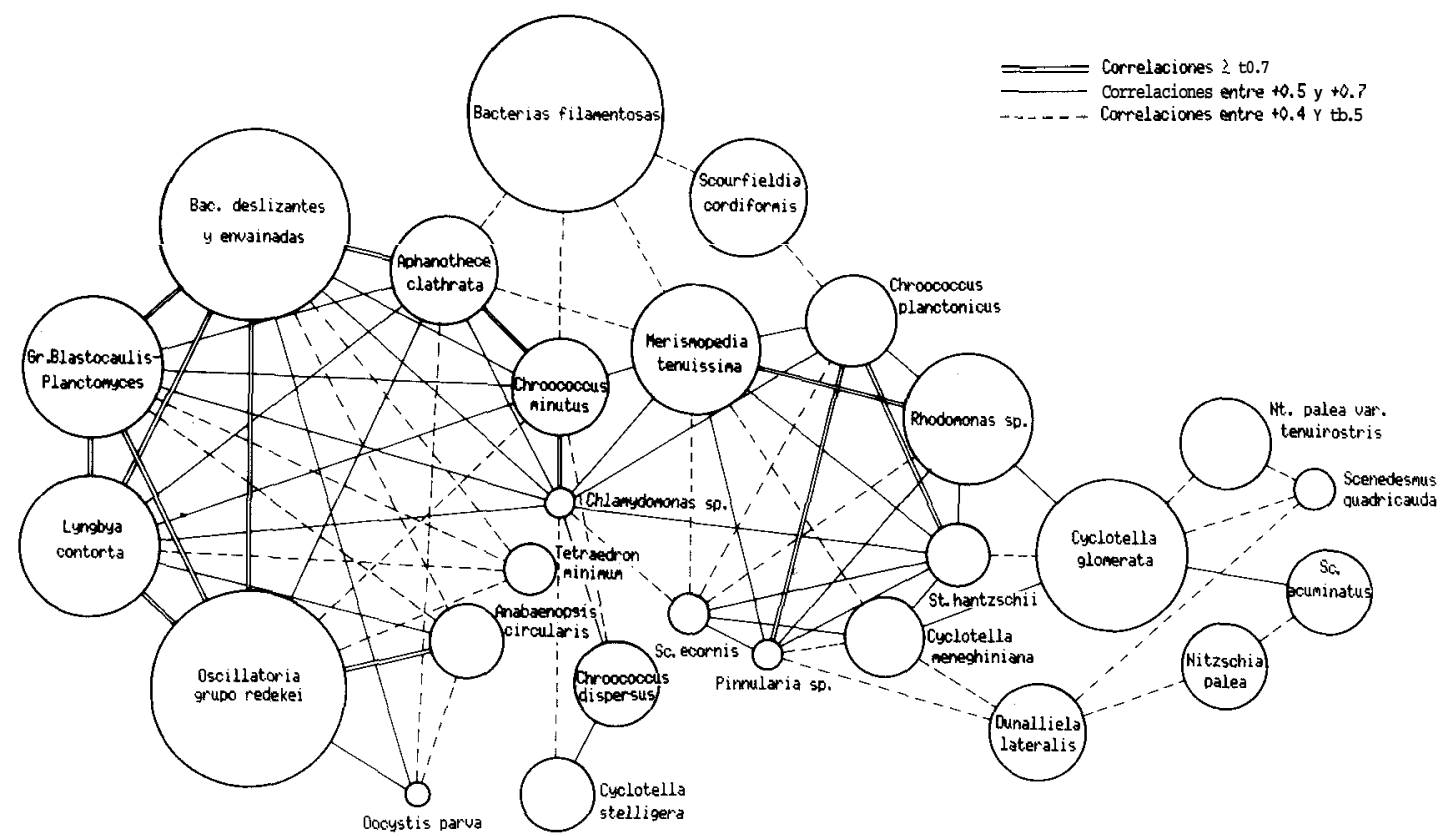

Figura 5.- Agrupación de las especies del fitoplancton por sus correlaciones. Cada especie está representada por un circulo cuyo diámetro es proporcional al logaritmo de su densidad de población, situandose según su coeficiente de correlación respecto a las demás especies. Solo se han considerado las correlaciones mayores de +0.4 .

Clustering of phytoplankton species by their correlations. Each species is represented by a circle whose diameter is proportional to the logarithm of their population density, and it is placed according to its correlation coefficients between the other species. Only correlations over +0.4 have been considered.

presencia, en el plancton de la Albufera, va acompañada del desarrollo masivo de vacuolas gaseosas distribuídas por todo el filamento. o con disposición terminal. Las especies de Cianobacterias son las más citadas en trabajos sobre la soprabiedad de las aguas, pues en su mayoría son organismos indicadores de aguas contaminadas y eutróficas, con mucha materia orgánica y altas temperaturas. Concretamente el género Lyngbya se caracteriza por desarrollarse e $\mathrm{n}$ aguas con índice de saprobiedad alto y algunas de sus especies se citan como tóxicas para muchos animales (Palmer, 1959) e incluso para el hombre, como es el caso de L. contorta, también muy abundante en la Albufera.

El grupo de las Cloroficeas, aunque numéricamente no puede competir con las cianobacterias, es el que presenta mayor variedad específica, distribuyéndose sus integrantes en enjambres de especies congenéricas, principalmente de los géneros Scenedesmus y Monoraphidium, representados por 10 y 7 especies respectivamente. En cuanto a las diatomeas, encontramos preferentemente, en la Albufera,las de tipo céntrico, con preponderancia del género Cyclotella que llega a alcanzar cantidades del orden de 500.000 individuos/ml. Respecto a las diatomeas pennadas, el género Nitzschia es el más importante, siendo especialmente abundantes $\mathrm{N}$. palea y su variedad tenuirostris, ambas muy comunes en aguas eutróficas.

Es interesante constatar el hecho de que especies de distribución tropical, poco frecuente en nuestro país, tales como Anabaenopsis circularis, dada la progresiva eutrofización de las aguas litorales, han extendido su área de distribución encontrándose actualmente en la Albufera.

El índice de diversidad del fitoplancton en la Albu. fera (Talba II) presenta valores bajos en los meses de verano, destacando el mínimo de 1,88 bits/individuo correspondiente a la estacicín 5 durante el mes de julio, debido al pronunciado "bloom" de Oscillatoria grupo redekei. Tras la renovación de agua que tiene lugar durante el mes de septiembre, por la apertura de las compuertas, el índice de diversidad experimentó un importante aumento en todas las localidades; así por ejemplo, la estación 5 que presentaba el antedicho mínimo en julio se alcanzaba en septiembre un máximo de 3,61 bits/individuo. No obs tant, en la estación 7 el índice experimentó un ligero descensoen aquel mes debido aunfuertecrecimiento de Cyclotella glomerata. 
Tabla III. Especie, mas frecuentes del fitoplancton de lá Vlbufera de \alencia, indicando su presencia (P) como porcentaje de muestras en las que estaban presente, su frecuencia $(F)$ en \% respecto del total de individuos del fitoplancton, su densidad niedia en aquellas muestras en las que está presente (11)、 su densidad máxima (MX), ambas en miles de individuos/mil, especificándose el mes y el punto de muestreo de dicho máximo.

Las especies señaladas con corresponden a las representadas en la fig. 5.

Most frequent species of the Albufera of Valencia phvtoplankton, indicating sts occurrence $(\mathrm{P})$ as percentage of sambles m uhich each occurred, its frequency (F)as percentage relative to total phytoplankton, dnd its mean density in those samples in which it occurred meandensity (M) and its maxımum density (MAX), thousands of jndividuals/ml, giiing the month and sampling station in which this maxımum is recorded.

Species with ${ }^{*}$ correspond to those presented in fig. 5

\begin{tabular}{|c|c|c|c|c|c|}
\hline CIAMOBACTERIAS & $P \%$ & $F \%$ & $\mathrm{~N}$ & NAX & \\
\hline * Lyngbya contorta & 96 & 2.61 & 2'3.4 & 94.2 & $\operatorname{Sp} 8$ \\
\hline Oscillatoria aghardii & 46 & 4.00 & 0.8 & 1.2 & \\
\hline * Oscillatoria grupo redekei & 100 & 29.85 & 256.51 & 057.9 & JI 5 \\
\hline Oscillatoria sp. ol. & 25 & 0.03 & 0.9 & 2.6 & Sp 4 \\
\hline * Merismodedia tenuissina & 100 & 1.64 & 14.1 & 66.5 & Sp 8 \\
\hline Merismopedia ourictata & 42 & 0.05 & 0.9 & 1.7 & \\
\hline * Chroococcus dispersus & 75 & 0.19 & 2.1 & 5.7 & \\
\hline * Chroococcus olanctonicus & 58 & 0.19 & 2.8 & 8.6 & \\
\hline * Chroococcus minutus & 79 & 0.41 & 4.5 & 9.5 & Sp \\
\hline * Anobaenoosis ciroularis & 58 & 0.13 & 1.9 & 4.6 & $J 1$ \\
\hline Nicrocyst1s pulverea & 38 & 0.04 & 0.9 & 2.3 & $\sqrt{1}$ \\
\hline Spirulina albida & 21 & 0.02 & 0.6 & 1.7 & \\
\hline * Aphanothece clathrata & 67 & 0.55 & 7.1 & 14.5 & J1 \\
\hline $\begin{array}{c}\text { Aphanothece sp. } \\
\text { DIATOMEAS }\end{array}$ & 33 & 0.09 & 2.2 & 4.6 & .11 \\
\hline * Cyclotella meneghiniana & 54 & 0.16 & 2.5 & 5.2 & I1 7 \\
\hline * Crclotella glomerata & 75 & 4.02 & 46.0 & 501.1 & So 7 \\
\hline Cyclotella striata & 8 & $0.0^{\prime} 3$ & 2.7 & 5.2 & $\mathrm{Sp}$ \\
\hline * Eyclotella stelliqera & 21 & 0.11 & 4.4 & 10.4 & Sp \\
\hline Cyclotella sp. ol. & 88 & 0.71 & 7.0 & 31.0 & Sp \\
\hline * Stephanodiscus hantzschii & 50 & 0.08 & 1.4 & 3.5 & $S_{p} 5$ \\
\hline * Nitzsohia polea & 67 & 0.30 & 3.8 & 10.4 & Sp \\
\hline * Nt. palea var, tenuirostris & 50 & 0.22 & 3.8 & 10.1 & Sp \\
\hline Nitzschia lorroissima & 29 & 0.01 & 0.4 & $1 . ?$ & $\mathrm{~N} 2$ \\
\hline Nitzschiэ intermedia & 33 & 0.10 & 2.7 & 6.4 & $S p$ \\
\hline Coconeis placentuls & 13 & 0.02 & 1.1 & 2.6 & 112 \\
\hline $\begin{array}{r}\text { * Pinnularia so. } \\
\text { 日GGNEC }\end{array}$ & 8 & 0.02 & 1.5 & 2.9 & 50 \\
\hline Euglena spl. & 54 & 0.13 & 2.0 & 5.2 & Hu \\
\hline $\begin{array}{l}\text { Euglena sD2. } \\
\text { CLOROFICEAS }\end{array}$ & 17 & 0.01 & 0.6 & 1.2 & \\
\hline Ankistrodesmus grocillis & 17 & 0.33 & 1.4 & 2.6 & \\
\hline Ankistrodesmus bibraianus & 29 & 0.03 & 0.8 & 1.7 & \\
\hline Monoraphidium griffithii & 29 & 0.02 & 0.5 & 1.2 & Nu \\
\hline Monoraphidiun ousilun & 71 & 0.24 & 2.9 & 13.8 & So 7 \\
\hline Monoraphidium contortur & 25 & 0.02 & 0.5 & 1.7 & $S_{p} 1$ \\
\hline Monor aphidium minutuil & 54 & 0.18 & 2.8 & 6.4 & Wo 7 \\
\hline Monoraphidium braunil & 46 & 0.08 & 1.4 & 5.7 & \\
\hline
\end{tabular}

Monoraphidiun conwolutum

* Tetraedron minimu

Chodatella sp.

* Docystis parva

Actinastrum hantzsohi

Actinastrum sp.

Chlamydomanas kvildensis

* Chlamydomonas sp.

* Scourfieldia cordifornis

* Dunalliela lateralis

kirctmeriella obesa

Coelastrum microporum

Chlorogoniun elongatum

chlorogonium minutum

Chlorogonium fusiforme

Golenkinia radiata

Fediastrum boryanum

Scenedesmus longispina

* Ccenedesmus quadricauda

* Ccenedesmus acuminatus

Sc. acuminatus f. tortuosus

* Scenedesmus ecorne

Ccenedesmus acutus

Sc. acutus f. altemprans

Scenedesmus peosens 1 :

Scenedesmus spanosus

scenedesmus granulatus

Ccenedesmus sp.

DINOFAGEAOOS

Gumnodinium lacustre CRIPTOFICEAS

* Rhodomonas so.

Chroomonas $\mathrm{sp}$.

$$
\text { BACTERIAS }
$$

* Ir. Blastocalis-Planctomeces

+ Bac. deslizantes y envainadas

Pasteilria rimasa

+ Bacterias tilamentosas

\begin{tabular}{|c|c|c|c|c|}
\hline$P \%$ & $F \vartheta$ & $\mathrm{N}$ & MAX & \\
\hline 29 & 0.05 & 1.6 & 3.5 & 504 \\
\hline 38 & 0.05 & 1.2 & 2.6 & $\$ 16$ \\
\hline 75 & 0.14 & 1.6 & 4.R & 515 \\
\hline 8 & 0.01 & 1.2 & 2.3 & 515 \\
\hline 54 & 0.29 & 4.6 & 10.4 & Nu 6 \\
\hline 71 & 0.05 & 0.6 & 1.7 & I1 8 \\
\hline 17 & 0.01 & 0.7 & 1.2 & Hu 1 \\
\hline 13 & 0.02 & 1.5 & 2.3 & Sp 7 \\
\hline 92 & 0.86 & 8.1 & 32.7 & $S_{0} 1$ \\
\hline 79 & 0.32 & 3.4 & 7.8 & 517 \\
\hline 71 & 0.15 & 1.8 & 3.5 & Sp S \\
\hline 33 & 0.05 & 1.4 & 3.5 & $5 p$ \\
\hline 21 & 0.01 & 0.6 & 1.7 & II 5 \\
\hline 8 & 0.02 & 1.6 & 2.3 & 118 \\
\hline 17 & 0.01 & 0.7 & 1.2 & Wv 6 \\
\hline 17 & 0.02 & 1.1 & 1.7 & Wu 4 \\
\hline 21 & 0.01 & 0.6 & 1.2 & 115 \\
\hline 13 & 0.02 & 1.2 & 1.2 & Sp 4 \\
\hline 13 & 0.02 & 1.7 & 2.6 & Hu $b$ \\
\hline 96 & 0.19 & 1.7 & 3.5 & So 6 \\
\hline 83 & 0.16 & 1.7 & 5.2 & II 5 \\
\hline 46 & 0.03 & 0.6 & 1.2 & 55 \\
\hline 67 & 0.16 & 2.0 & 10.4 & sp 5 \\
\hline 21 & 0.02 & 0.9 & 1.7 & $5 \mathrm{p} 1$ \\
\hline 13 & 0.02 & 1.7 & 1.7 & 807 \\
\hline 29 & 0.02 & 0.6 & 1.7 & 507 \\
\hline 29 & 0.08 & 2.3 & 5.2 & 505 \\
\hline 17 & 0.02 & 1.2 & 2.6 & Sp 3 \\
\hline 38 & 0.04 & 0.9 & 1.7 & 56 \\
\hline 88 & 1.30 & $12 . ?$ & 37.9 & 503 \\
\hline 17 & 0.09 & 4.5 & 10.4 & 504 \\
\hline 96 & 2.53 & 22.7 & $10 \mathrm{c}, \mathrm{2}$ & 111 \\
\hline 100 & 23.60 & 202.8 & 650.9 & 114 \\
\hline 33 & 0.06 & 1.4 & 3.5 & Nu 5 \\
\hline 100 & 27.27 & 234.3 & 555.7 & $\operatorname{sog}$ \\
\hline
\end{tabular}



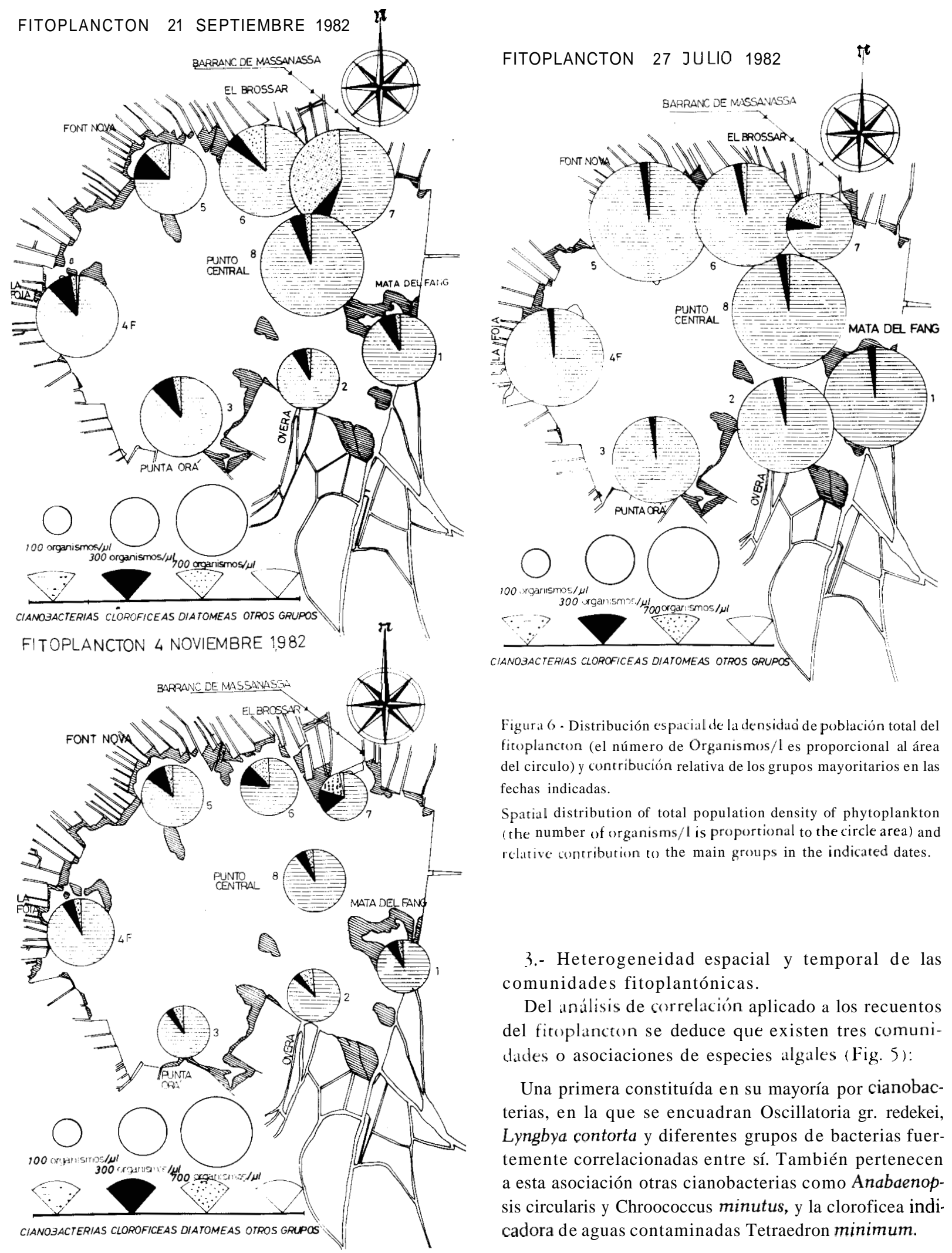

Figura 6. Distribución espacial de la densidado de población total del fitoplancron (el número de Organismos/l es proporcional al área del circulo) y contribución relativa de los grupos mayoritarios en las fechas indicadas.

Spatial distribution of total population density of phytoplankton (the number of organisms/l is proportional to the circle area) and relative contribution on the main groups in the indicated dates.

3.- Heterogeneidad espacial y temporal de las comunidades fitoplantónicas.

Del análisis de correlación aplicado a los recuentos del fitoplancton se deduce que existen tres comunidades o asociaciones de especies algales (Fig. 5):

Una primera constituída en su mayoría por cianobacterias, en la que se encuadran Oscillatoria gr. redekei, Lyngbya contorta y diferentes grupos de bacterias fuertemente correlacionadas entre sí. También pertenecen a esta asociación otras cianobacterias como Anabaenopsis circularis y Chroococcus minutus, y la cloroficea indicadora de aguas contaminadas Tetraedron minimum. 
La segunda comunidad constituída por Stephanodiscus bantzscbii, Chroococcus planctonicus, Scenedesmus ecornis, Pinnularia sp.. Merismopedia tenuissima y Rhodomonas sp.

La tercera comunidad consta fundamentalmentede

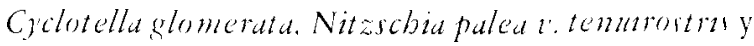
especies de, Scenedesmus

Además, estas tres asociaciones se hallan relacionadas ligeramente entre sí, pues existen especies que se incluyen simultáneamente en más de una de ellas, si bien las tres asociaciones se definen lo suficiente.

Como consecuencia de la mencionada estructuración del fitoplancton se pueden distinguir en la Albufera tres zonas principales:

1) La zona del Barranc de Massanassa (est. 7) que destaca claramente como diferente de todas la demás, caracterizada por el grupo de asociación tercero y por ser la localidad donde se encuentran más especies de euglenales.

2) El sector Norte de la Albufera al Oeste del Barranc de Massanassa (ests. 5 y 6) presentando muchas afinidades con la primera zona descrita, pero que podríamos considerar caracterizado por la comunidad segunda.

3) El resto de la Albufera, donde predomina absolutamente el primer grupo de asociación, grupo que en realidad domina en todo el lago, pero que en esta zona totaliza más del $90 \%$ de la población.

La figura 6 registra la diferente distribución de los grupos algales en los distintos puntos e ilustra acerca de la gran dominancia de las cianobacteria. Sin embargo, en la estación 7 su dominancia nunca excede el $75 \%$. Puede observarse también la distinta variación en el tiempo de las tres estaciones del Norte (ests. 5, 6 y 7) con respecto a las demás, que podríamos resumir en dos puntos:

1) De julio a septiembre se observa una disminución de la abundancia de cianobacterias que dejan de totalizar el $90 \%$ del fitoplancton, disminución que resulta especialmente marcada en las estaciones del Norte $(5,6$ y 7$)$, donde son reemplazados por diatomeas. Sin embargo, en las restantes localidades la disminución no es tan manifiesta, contrarestando con un aumenro de las cloroficeas.

2) De Septiembre a Noviembre sucede lo inverso, ya que en las estaciones 1, 2, 3, 4 y 8 disminuyen las clorofíceas en favor de un aumento de las diatomeas, mientras que en las estaciones del Norte $(5,6$ y 7 ) se tiende a experimentar un descenso en el porcentaje de las diatomeas en favor de las cloroficeas, muy claramente marcado en la estación 7.

Todo esto podría significar una respuesta a entradas de agua en la laguna, con el consiguiente descenso de la mineralización y aumento de la turbulencia, condiciones estas favorecedoras del crecimiento de las diatomeas. En Septiembre, con apertura de las compuertas que regulan el nivel del lago, acorde con el cultivo del arroz, se produce un flujo de agua cuya mayor intensidad radica en el sector Norte de la laguna, lo que consecuentemente induce un crecimiento de las diatomeas (est. 7) y de las cloroficeas (est. 5 y 6). En Noviembre, tras las inundaciones catastróficas del 20 de Octubre de 1982, que supusieron una fuerte entrada de agua por la zona Sur, se aprecia también un aumento de las diatomeas, esta vez en las estaciones 2 y 3 , que fueron las más afectadas por dicha entrada de agua.

\section{CONSIDERACIONES FINALES}

La Albufera de Valencia presenta una densidad fitoplanctónica muy elevada que supera siempre los 200.000 individuos $/ \mathrm{ml}$ y llega, en muchas ocasiones, a superar el millón de ind./ml (el máximo medido en este estudio es de 1.750 .000 ind./ $\mathrm{ml}$ ). Su biomasa es en la mayoría de las ocasiones superior a $100 \mathrm{~g} / \mathrm{m}^{3}$ y en varias localidades alcanza valores superiores a los $200 \mathrm{~g} / \mathrm{m}^{3}$ (el máximo medido es de $276 \mathrm{~g} / \mathrm{m}^{3}$ ).

La clorofila $a$ es siempre superior a $100 \mathrm{mg} / \mathrm{m}^{3} \mathrm{y}$ la mayoria de localidades presenta valores superiores a $400 \mathrm{mg} / \mathrm{m}^{3}$ (el máximo medido es de $646 \mathrm{mg} / \mathrm{m}^{3}$ ). En el estudio del fitoplancton de los embalses españoles (Margalef, 1977) se llegó a la conclusión de que aquellos embalses cuya eutrofia era más extrema podían llegar a soportar poblaciones del orden de 200.000 ind./ $\mathrm{ml}$ que corresponderían a $100 \mathrm{mg} / \mathrm{m}^{3} \mathrm{de}$ clorufila $a$. Barica (1980) define los lagos hipertróficos como el último estadío de la eutrofización y se refiere a los lagos hipertróficos extremos como aquellos en que se sobrepasan los $100 \mathrm{mg} / \mathrm{m}^{i}$ de clorofila $\boldsymbol{a}$ o $\operatorname{los} 100 \mathrm{~g} / \mathrm{m}^{2}$ de biomasa algal. La Albufera sobrepasa en mucho estos valores y los correspondientes a los lagos y embalses, situandose por estos y otros parámetros entre los sistemas más eutróficos del mundo.

También señalaremos que el plancton vegetal que ocupa la Albufera en la actualidad se diferencia en 
mucho del que la poblara años atras, cuando no había alcanzado tan alto grado de eutrofia, entonces la flora planctónica dominante estaba constituída principalmente por diatomeas (Pardo, 1942), mientras que ahora aquellas poblaciones han sido reemplazadas en gran medida por especies de cianobacterias. El avance en esta tendencia supone la continua desaparición de especies, compensada por la aparición de solo unas pocas que se hacen dominantes, todo ello de forma paralela a como se avanza en el vertido incontrolado de deshechos al lago.

Las cianobacterias dominan el fitoplancton de la Albufera, del que llegan a constituir hasta el 95\% del número de individuos, excepto e n las estaciones de la zona Norte, que albergan comunidades con un mayor número de diatomeas que llegan incluso a superar a las cianobacterias en algún momento. Esta zona se diferencia también del resto de la laguna en ciertos caracteres químicos del agua, resultando un dato muy aparente su tendencia a un mayor grado de salinidad (Oltra y Miracle, 1984). Entre las causas para estas diferencias, cabe destacar el hecho de que las estaciones del Norte son puntos cercanos a los aportes de agua procedente de la cuenca del Turia, mientras que las zonas Oeste y Sur están más relacionadas con las procedentes del Jucar.

\section{AGRADECIMIENTOS}

Este trabajo ha sido realizado gracias a las facilidades prestadas por el Excmo. Ayuntamiento de Valencia y a la subvención otorgada por la CAICYT (proyecto $n^{0} 915 / 81$ ).

\section{BIBLIOGRAFIA}

Barica, J. 1980. Why hypertrophic Ecosystems? In Hypertrofic Ecosystems (Barica,J. y L.R. Mur eds.) D H 2.Junk. The Hague.348 págs.

Blanco, C. 1975. Estudio de la contaminación de la Albufera de Valencia y de los efectos de dicha contaminación sobre la fauna y la flora del lago. Tesis doctoral. Universidad de Valencia,

Dafauce, C. 1975. La Albufera de Valencia. Un estudio piloto.Monografias ICONA, 4 Madrid. 127 págs.

Lorenzen, C.J. 1967. Determination of chlorophyll and pheopigments: spectrophotometric equations. Limnol. Oceanogr., 12: 343-348.

Margalef, R.; Planas, D.; Armengol, J.; Vidal, A.; Prat, N. Guiset, A.; Toja, J. y Estrada, M. 1977. Limnología de los embalses españoles Ministerio de Obras Públicas. Madrid. 452 pp. Margalef, R. 1980. Ecología Omega. Barcelona 951 pp.

Oltra, R. y M.R. Miracle. 1984. Comunidades zooplanctónicas de la Albufera de Valencia Limnetica 1 5I-61)

Palmer, M.C. 1959. Algas en abastecimientos de agua, Interamericana. MEXICO. 91 págs.

Pardo, L.1942. La Albufera de Valencia. Estudio Limnográfico, biológico, económico y antropológico. Bol. Inst. Forestal Inv. y Exper. 163 págs

Planas, M.D. 1973.Composición, ciclo y productividad del fitoplancton del lago de Bayoles. Oceología Aquática, 1: 3-106 Rosello, V.M. 1979. Els espais albuferencs del País Valencia. Acta Geología Hispánica, $14:$ 487-493.

Shoaf, W.T. \& B.W. Lium 1976. Improved extraction of chlorophyll a and b from algae using dimethyl-sulphoxide. Limnol. Oceanogr. 21: $926 \cdot 928$.

Strickland, J.D.H.\& T.H. Parsons 1972. A practica1 handbookof seawaters analyses. 2nd ed. Bull. Fish. Res. Bd. Canadá. 167 págs.

Toja, J . 1980.Limnología del embalse de La Minilla durante 1976. I. Ciclo del fitoplancton en relación con los factores del medio. Oceanología Aquática, 4: 71-88.

Vollenweider, R.A. 1974. A manual on methods for measuring primary production in aquatic environinents. IBP Handbook No. 12 Blackwell Oxford. 225 pp. 